\title{
Hydrophobic Functionalization of Polyacrylic Acid as a Versatile Platform for the Development of Polymer Lipid Nanodisks
}

\author{
Nathaniel Z. Hardin, Thirupathi Ravula, Giacomo Di Mauro, \\ and Aypalusamy Ramamoorthy*
}

\begin{abstract}
Polymer nanodisks have shown great potential as membrane mimetics that enable the study of functional membrane protein structural biology and also have a wider application in other fields such as drug delivery. To achieve these research goals, the ability to have a cheap, simple, fully customizable platform for future nanodisks technology applications is paramount. Here, a facile functionalization of polyacrylic acid (PAA) with varying hydrophobic groups that form nanodisks at different sizes is successfully demonstrated. The study shows that the choice of hydrophobic group can have a noticeable effect on the polymer solubilization properties and polymer-induced perturbation to the encased lipid bilayer. Due to this robust, tunable chemical synthesis method, PAA is an exciting platform for the future optimization of the hydrophobic, hydrophilic, or direct purposed functionalizations for polymer nanodisks.
\end{abstract}

(diisobutylene maleic acid co-polymer, ${ }^{[14]}$ DIBMA, and polymethacrylate $\left.{ }^{[13]}\right)$ have been shown to form nanodisks; however there has been no systematic investigation comparing the effects of varying the hydrophobic functional groups used in the formation of nanodisks. In this study, we employed a simple and robust modification method to vary the hydrophobic groups on a commercially available low molecular weight polyacrylic acid (PAA) which allowed us to observe how alkylPAA affected the formation, stability, and other properties of nanodisks.

In the past hydrophobically modified PAAs were shown to act as amphipols which have the ability to form pores in a membrane and solubilize MPs. ${ }^{[15,16]}$ We

Polymer nanodisks have emerged as a new native-like membrane mimetic in recent years and are routinely being used for the study of membrane proteins (MPs). ${ }^{[1-3]}$ A major advantage of using polymers is that they can directly extract MPs from their native environment as shown by the styrene-maleic acid copolymers (SMA). ${ }^{[1,2,4-6]}$ Despite the great potential, polymer nanodisks have suffered from some major drawbacks due to the intrinsic chemical properties of SMA inherent to its hydrophobic and hydrophilic units. ${ }^{[2,7]}$ Several studies have already shown an enhancement of SMA's stability by the modification of hydrophilic functional units. ${ }^{[7-12]}$ The most common hydrophobic group used in SMA polymer nanodisks is the styrene moiety. It is known that styrene has a strong absorption in the UV region that can interfere with various biophysical techniques, and can also have nonspecific interactions with other aromatic groups from the protein. ${ }^{[13,14]}$ Recently, two styrene-free polymers

N. Z. Hardin, Dr. T. Ravula, G. Di Mauro, Prof. A. Ramamoorthy

Biophysics Program and Department of Chemistry

Biomedical Engineering

Macromolecular Science and Engineering

University of Michigan

Ann Arbor, MI 48109-1055, USA

E-mail: ramamoor@umich.edu

The ORCID identification number(s) for the author(s) of this article can be found under https://doi.org/10.1002/smll.201804813.

DOI: $10.1002 / \mathrm{smll} .201804813$ hypothesized that modifying a short chain PAA with relatively short alkyl groups (4-6 carbons), as compared to the long alkyl groups ( $>8$ carbons) used in the formation of amphipols, ${ }^{[17]}$ would produce an amphiphilic polymer with the ability to form nanodisks. To test this hypothesis, we used a low molecular weight $\left(M_{\mathrm{w}}=1800 \mathrm{Da}\right)$ PAA as the starting material. Different hydrophobic groups (butyl, pentyl, hexyl, and neopentyl) were chosen to functionalize PAA. To achieve this, we applied a simple condensation reaction scheme to PAA using $N$-(3-dimethylaminopropyl)- $N$-ethylcarbodiimide hydrochloride as our coupling reagent and the appropriate alkyl amine as the reactant (Figure 1a; Supporting information). The resulting polymer was characterized using Fourier transform infrared spectroscopy (FT-IR), proton-nuclear magnetic resonance spectroscopy $\left({ }^{1} \mathrm{H}-\mathrm{NMR}\right)$, and carbon-13-cross polarization magic angle spinning $\left({ }^{13} \mathrm{C}\right.$-CPMAS) solid-state NMR experiments. FT-IR spectra show amide stretching frequency $\left(\approx 1640 \mathrm{~cm}^{-1}\right)$ for the products confirming the successful completion of the coupling reaction (Figure $1 \mathrm{~b}$ ). This was further confirmed by the carbonyl carbon resonance $(\approx 185-180 \mathrm{ppm})$ and the appearance of new peaks in the aliphatic region $\left(\mathrm{CH}_{2} \approx 32 \mathrm{ppm}\right.$, $\mathrm{CH}_{3} \approx 17 \mathrm{ppm}$, and quaternary $\mathrm{C} \approx 54 \mathrm{ppm}$ ) in ${ }^{13} \mathrm{C}$-CPMAS NMR spectra of the synthesized polymers as compared to PAA starting material (Figure 1c). ${ }^{1} \mathrm{H}$-NMR spectra were used to estimate the extent of functionalization by integration (Figure S1, Supporting Information) to be $\approx 40-50 \%$, within the range of optimal hydrophobic to hydrophilic ratio as seen previously for nanodisks. ${ }^{[13]}$ 

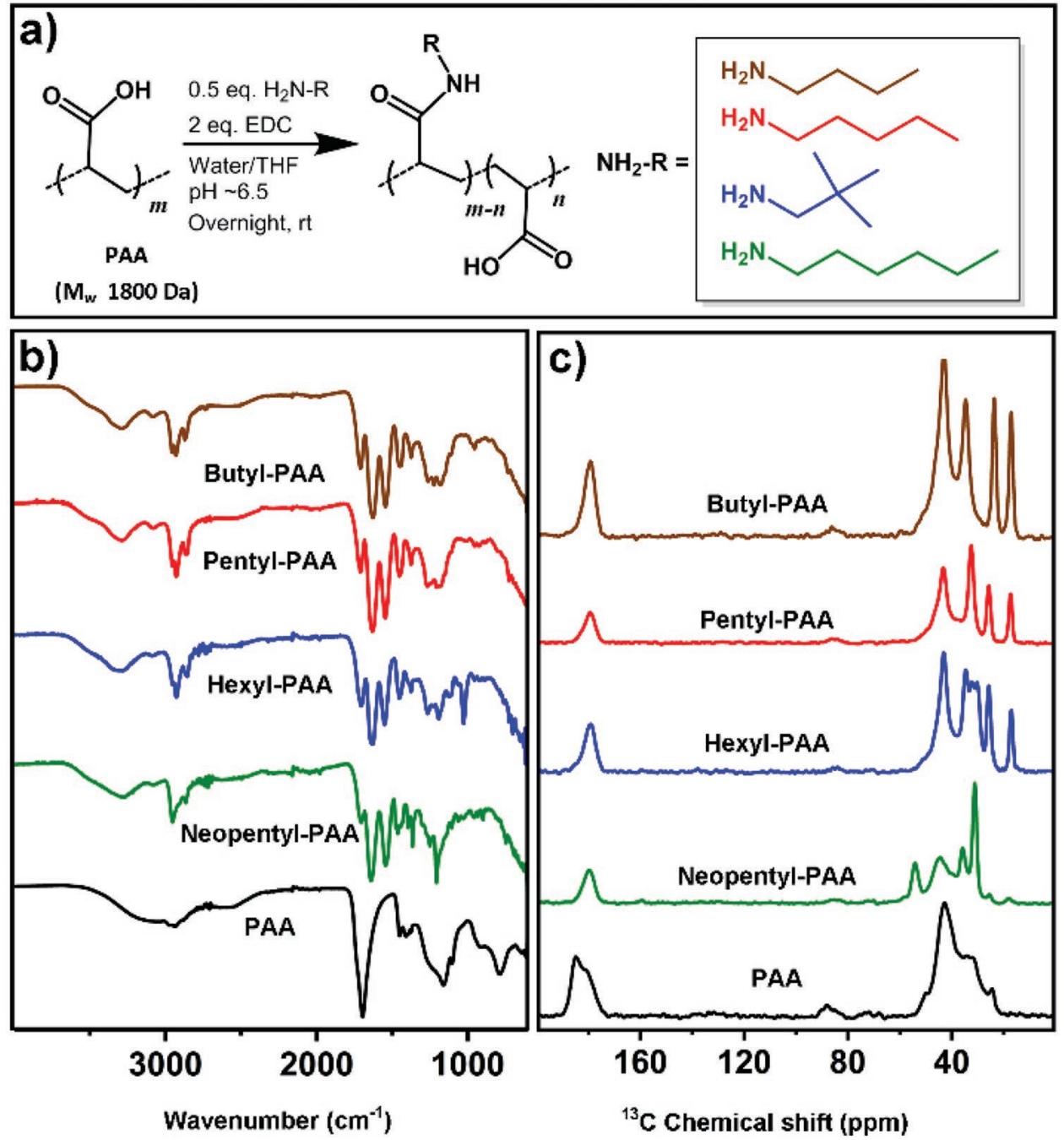

Figure 1. Synthesis and characterization of PAA polymers. a) General reaction schematic of PAA functionalization. b) $\mathrm{FT}-\mathrm{IR}$ and $\mathrm{c}){ }^{13} \mathrm{C}-\mathrm{CPMAS}$ solid-state NMR spectra of functionalized alkyl-PAA.

The resulting polymers were mixed with 1,2-dimyristoyl-snglycero-3-phosphocholine (DMPC) at different polymer:lipid weight ratios $(0.25: 1,0.5: 1,1: 1)$. Static light scattering (SLS) measurements were used to monitor the solubilization of DMPC multilamellar vesicles (MLVs). While the Pentyl-PAA, Neopentyl-PAA, and Hexyl-PAA were found to solubilize DMPC vesicles into small particles, the Butyl-PAA needed a much higher amount of polymer to achieve solubilization $(>1: 1)$ and therefore was not used in further studies (Figure S2, Supporting Information). In order to investigate the size distribution of the polymer nanodisks, we used size exclusion chromatography (SEC) and dynamic light scattering (DLS; Figure 2). Polymers were mixed with DMPC MLVs at appropriate weight ratios and then incubated overnight at $32{ }^{\circ} \mathrm{C}$. SEC chromatograms showed the presence of two peaks: nanodisks eluted within the region of 9-12 $\mathrm{mL}$ whereas the free polymer eluted at $15-20 \mathrm{~mL}$. All three polymers showed size tunability by varying the polymer:lipid ratio. We concluded this by the observation of a shift in the retention volume of the nanodisk's peak and no shift in the retention volume of the free polymer peak in the SEC profiles. Neopentyl-PAA and Pentyl-PAA nanodisks showed a major increase in the intensity of the polymer peak only at high polymer:lipid ratios. Hexyl-PAA showed the presence of new peaks ( 8.5 and $14.3 \mathrm{~mL})$ and no shift in the nanodisk's peak at high polymer:lipid ratio, suggesting a saturation point in the polymer:lipid ratio needed to form nanodisks was reached (Figure 2a-c). The corresponding DLS profiles showed size variation as a function of polymer:lipid ratio (Figure $2 \mathrm{~d}-\mathrm{f}$, Table 1) and were in good agreement with SEC observations similar to previous observations using SMA derivatives. ${ }^{[7,8]}$

The polymer nanodisks were further characterized using transmission electron microscopy (TEM). The TEM images of the polymer nanodisks with differing weight ratios clearly showed the presence of disk shaped particles of varying sizes complementary to SEC and DLS results (Figure $2 \mathrm{~g}-0$ ). These results confirmed that these polymers can form nanodisks and the size of nanodisks can be controlled by varying the polymer:lipid ratio.

The stability of the nanodisks to the presence of divalent metal ions and $\mathrm{pH}$ were tested using SLS. Akyl-PAA polymers 

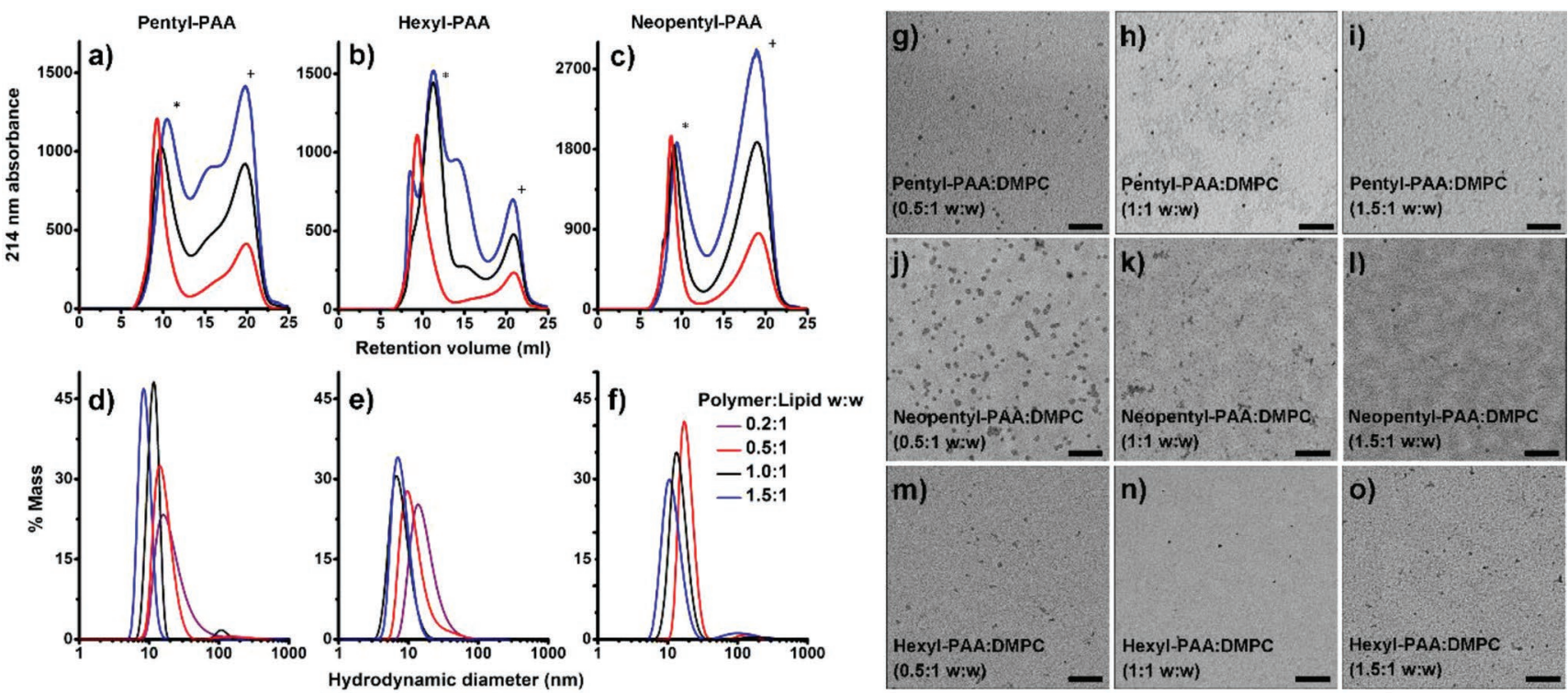

Figure 2. Characterization of PAA polymer nanodisks. a-c) SEC and $d-f$ ) DLS profiles of nanodisks prepared at the indicated polymer:lipid ratios. $(*)$ denotes nanodisks fractions collected and $(+)$ denotes free polymer fraction. TEM images $g-0$ ) of samples prepared at the specified polymer:lipid ratio; scale bar represents $200 \mathrm{~nm}$.

were found to have very similar stability properties toward $\mathrm{pH}$ and metal ions as compared to SMALP due to the presence of carboxylic groups as the hydrophilic functional group (Table 1, Figures S3-S5, Supporting Information). Due to the absence of aromatic moieties, the akyl-PAA polymers showed no absorbance at $254 \mathrm{~nm}$ (Figure S6, Supporting Information). The effect of polymer hydrophobic group on lipid bilayer properties was studied using differential scanning calorimetry (DSC) experiments. The DSC profiles of most polymer-DMPC-nanodisks showed a typical gel to liquid crystalline phase transition temperature $\left(T_{\mathrm{m}}\right)$ in the range of $\approx 25-27{ }^{\circ} \mathrm{C}$, which is very close to pure DMPC's $T_{\mathrm{m}}=24 \pm 1{ }^{\circ} \mathrm{C}$, suggesting the preservation of lipid dynamics upon the formation of nanodisks (Table 1, Figure S7, Supporting Information). Pentyl-PAA showed only a $1{ }^{\circ} \mathrm{C}$ change in the phase transition temperature between the low $(0.2: 1 \mathrm{w}: \mathrm{w})$ and high $(1: 1 \mathrm{w}: \mathrm{w})$ polymer:lipid ratios, signifying only a minor perturbation of the lipid bilayer. Neopentyl-PAA nanodisks were seen to have both a similar major transition temperature $\left(\approx 26^{\circ} \mathrm{C}\right)$ and a minor lower transition temperature at $\approx 19^{\circ} \mathrm{C}$. This observation may be interpreted as Neopentyl-PAA increasing the disorder of those lipids located close to the polymer-belt of the nanodisks. Hexyl-PAA at a low polymer:lipid ratio exhibited a similar behavior to that observed for Pentyl-PAA, whereas at a higher polymer:lipid ratio the transition temperature is shifted from $\approx 26$ to $\approx 22{ }^{\circ} \mathrm{C}$ and is significantly broadened. This change is likely due to a strong perturbation of the lipid bilayer by the longer alkyl chain.

Since the size of PAA polymer based nanodisks were easily tunable, we prepared macro-nanodisks and examined their ability to spontaneously align in the presence of a magnetic field. All three types of polymer macro-nanodisks were tested with the lowest polymer:lipid ratio possible (Table 1) using ${ }^{31} \mathrm{P}$ and ${ }^{14} \mathrm{~N}$ static solid-state NMR experiments. The ${ }^{31} \mathrm{P}$ NMR spectra were recorded at different temperatures ranging from 280 to $320 \mathrm{~K} .{ }^{31} \mathrm{P}$-NMR showed the appearance and disappearance of two main peaks at $\approx-1.5 \mathrm{ppm}$ and in the $\approx-12$ to $\approx-14 \mathrm{ppm}$ region as a function of temperature. The peak at $\approx-1.5 \mathrm{ppm}$ is due to the fast tumbling of isotropic nanodisks, whereas the peak at $\approx-12$ to $\approx-14 \mathrm{ppm}$ is indicative of macronanodisks with the lipid phosphate head groups aligned perpendicular to the magnetic field axis. Pentyl-PAA macro-nanodisks showed an isotropic peak $(\approx-1.5 \mathrm{ppm})$ at $280 \mathrm{~K}$. Partial alignment of the nanodisks was seen ( $\approx-12 \mathrm{ppm})$ at $285-290 \mathrm{~K}$, and complete alignment above $295 \mathrm{~K}$ (Figure 3a). Hexyl-PAA macro-nanodisks showed alignment similar to Pentyl-PAA macro-nanodisks, however, they required a higher temperature $(300 \mathrm{~K})$ to fully align, and at $320 \mathrm{~K}$ a small isotropic peak was observed. Neopentyl-PAA macro-nanodisks had similar characteristics of Pentyl-PAA macro-nanodisks at lower temperatures $(<310 \mathrm{~K})$ above which a large isotropic signal was observed

Table 1. Properties of alkyl-PAA-DMPC-nanodisks.

\begin{tabular}{lccccccc}
\hline Hydrophobic group & Polymer/lipid (w/w) & Size ${ }^{\mathrm{a})}[\mathrm{nm}]$ & $\mathrm{PDI}$ & Elution volume $[\mathrm{mL}]$ & $\mathrm{pH}$ stability) & $\begin{array}{c}\mathrm{Mg}^{2+}, \mathrm{Ca}^{2+} \\
\text { stability }\left[\times 10^{-3} \mathrm{M}\right]\end{array}$ & $\begin{array}{c}\mathrm{DSC} \text { transition } \\
\text { temperature }\left[{ }^{\circ} \mathrm{C}\right]\end{array}$ \\
\hline Pentyl & $0.2,0.5,1,1.5$ & $16,14,12,8$ & $1.7,0.25,0.11,0.14$ & $\mathrm{nd}, 9.3,9.7,10.5$ & $>6$ & $5.5,3.5$ & $26, \mathrm{nd}, 25, \mathrm{nd}$ \\
Hexyl & $0.2,0.5,1,1.5$ & $14,10,7,7$ & $1.0,0.63,0.31,0.27$ & $\mathrm{nd}, 9.3,11.3,11.3$ & $>6$ & 2,2 & $26, \mathrm{nd}, 22, \mathrm{nd}$ \\
Neopentyl & $0.3,0.5,1,1.5$ & $\mathrm{nd}, 17,13,10$ & $\mathrm{nd}, 0.39,0.23,0.27$ & $\mathrm{nd}, 8.7,9.1,9.5$ & $>6.5$ & $5.5,2$ & $28, \mathrm{nd}, 26, \mathrm{nd}$ \\
\hline
\end{tabular}

a)hydrodynamic diameter measured from DLS; ${ }^{b, c}$ Measured from SLS. nd denotes not determined. polydispersity index (PDI) from DLS. 

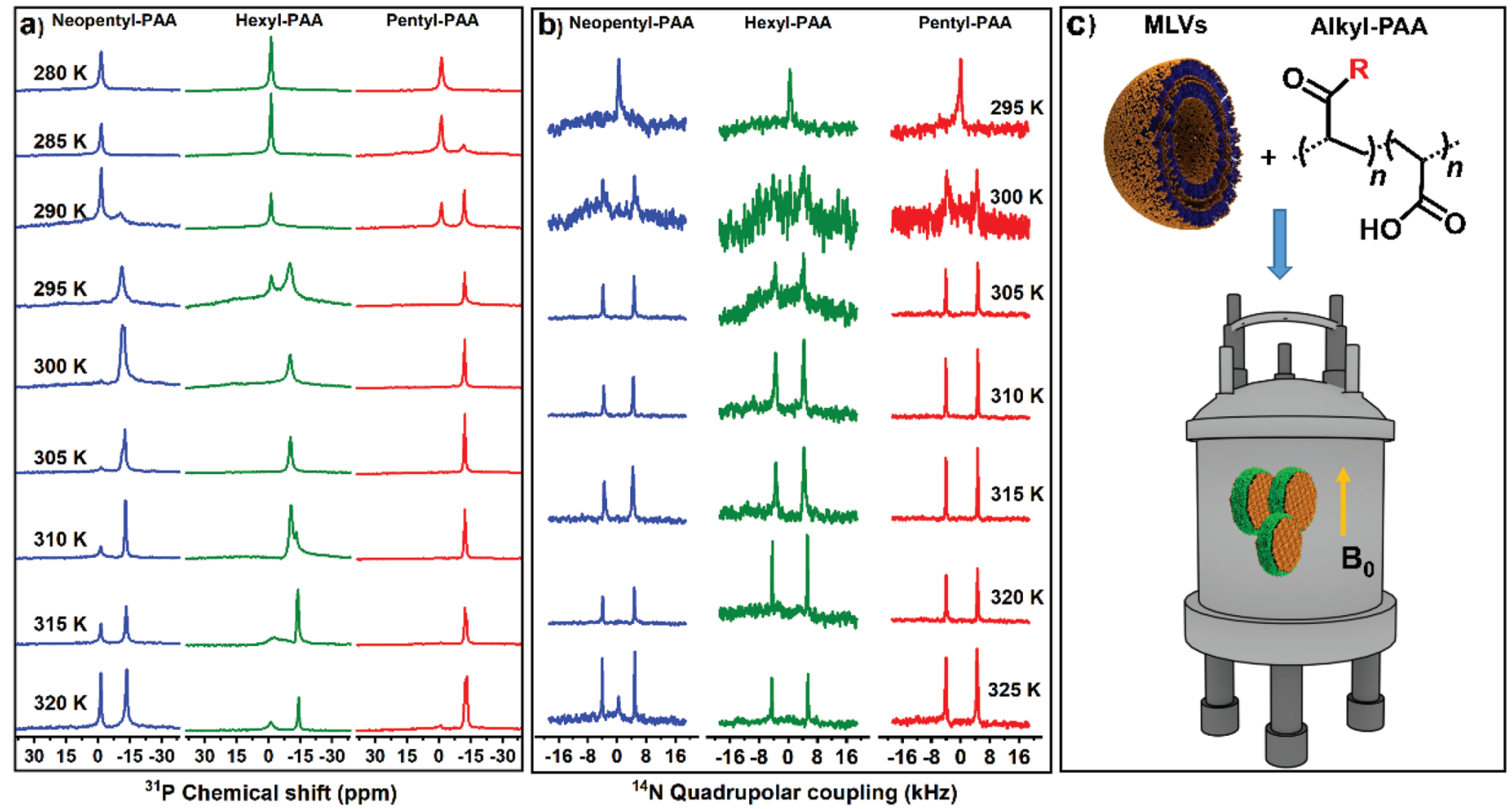

Figure 3. Magnetic alignment of PAA based macro-nanodisks. a) ${ }^{31} \mathrm{P}$ and b) ${ }^{14} \mathrm{~N}$ NMR spectra of macro-nanodisks prepared from NeopentylPAA:DMPC (0.3:1 w/w), Hexyl-PAA:DMPC (0.2:1 w/w), and Pentyl-PAA:DMPC $(0.2: 1 \mathrm{w} / \mathrm{w})$ at the indicated temperatures. c) Schematic representation of magnetic-alignment of macro-nanodisks.

suggesting less stability at higher temperature as compared to Pentyl-PAA. A similar trend was observed using ${ }^{14} \mathrm{~N}-\mathrm{NMR}$. The quadrupolar coupling of ${ }^{14} \mathrm{~N}$ nuclei is a direct measurement of the orientation of choline group $(\mathrm{C}-\mathrm{N}$ bond vector) relative to the magnetic field direction. While at low temperature ${ }^{14} \mathrm{~N}$ peaks were isotropic, at higher temperatures a quadrupolar coupling of $7-8 \mathrm{kHz}$ was observed further confirming the magnetic alignment of macro-nanodisks with lipid bilayer normal oriented perpendicular to the magnetic field (Figure 3b).

To demonstrate that these new polymers can be used for direct MP extraction from cellular membranes, we incubated the polymer with cell lysate following a published protocol ${ }^{[2]}$ (Supporting information). All three alkyl-PAA polymers showed similar efficacy as compared to SMALP as evident from the SDS-PAGE gel (Figure S8, Supporting information). These observations suggest that the hydrophobic modifications of PAA have the ability to extract MPs from their native environment.

In conclusion, we have successfully demonstrated the facile functionalization of PAA with differing hydrophobic groups that form nanodisks at varying sizes. By using a variety of techniques (SLS, DLS, SEC, TEM, DSC, and NMR) we have shown that the method of functionalization proved robust with multiple different sidechains that form nanodisks. This allowed us to systematically probe the important effects of differing hydrophobic functionalization on the intrinsic properties polymer nanodisks, which have yet to be seen. We show that the choice of hydrophobic group can have a noticeable effect on the polymer solubilization properties. Using these polymers we can control the extent of lipid bilayer perturbation which is vital for ensuring a more native like membrane environment. Due to this robust, tunable chemical synthesis method, PAA is an exciting platform for future optimization of the hydrophobic, hydrophilic, or direct purposed functionalization's for polymer nanodisks.

\section{Supporting Information}

Supporting Information is available from the Wiley Online Library or from the author.

\section{Acknowledgements}

This study was supported by the NIH (GM084018 to A.R.). The authors thank Professor Adam Matzger for fruitful discussion on polymer synthesis and Dr. Mukesh Mahajan for providing cells. The authors thank Polyscope Polymers for the generous gift of SMA-3000.

\section{Conflict of Interest}

The authors declare no conflict of interest.

\section{Keywords}

functionalization, magnetic alignment, membrane mimetic, nanomaterials, polymer nanodisks

Received: November 15, 2018

Revised: December 27, 2018

Published online: January 22, 2019 
[1] Z. Stroud, S. C. L. Hall, T. R. Dafforn, Methods 2018, 147, 106.

[2] S. C. Lee, T. J. Knowles, V. L. Postis, M. Jamshad, R. A. Parslow, Y. P. Lin, A. Goldman, P. Sridhar, M. Overduin, S. P. Muench, T. R. Dafforn, Nat. Protoc. 2016, 11, 1149.

[3] J. M. Dörr, M. C. Koorengevel, M. Schäfer, A. V. Prokofyev, S. Scheidelaar, E. A. W. van der Cruijsen, T. R. Dafforn, M. Baldus, J. A. Killian, Proc. Natl. Acad. Sci. USA 2014, 111, 18607.

[4] K. A. Morrison, A. Akram, A. Mathews, Z. A. Khan, J. H. Patel, C. Zhou, D. J. Hardy, C. Moore-Kelly, R. Patel, V. Odiba, T. J. Knowles, M.-u.-H. Javed, N. P. Chmel, T. R. Dafforn, A. J. Rothnie, Biochem. J. 2016, 473, 4349.

[5] Z. Hu, J. C. S. Ho, M. Nallani, Curr. Opin. Biotechnol. 2017, 46, 51.

[6] J. M. Dörr, S. Scheidelaar, M. C. Koorengevel, J. J. Dominguez, M. Schäfer, C. A. van Walree, J. A. Killian, Eur. Biophys. J. 2016, 45, 3.

[7] T. Ravula, N. Z. Hardin, S. K. Ramadugu, S. J. Cox, A. Ramamoorthy, Angew. Chem., Int. Ed. 2018, 57, 1342.

[8] T. Ravula, S. K. Ramadugu, G. Di Mauro, A. Ramamoorthy, Angew. Chem., Int. Ed. 2017, 56, 11466.

[9] T. Ravula, N. Z. Hardin, S. K. Ramadugu, A. Ramamoorthy, Langmuir 2017, 33, 10655.
[10] S. Lindhoud, V. Carvalho, J. W. Pronk, M. E. Aubin-Tam, Biomacromolecules 2016, 17, 1516.

[11] M. C. Fiori, Y. Jiang, G. A. Altenberg, H. Liang, Sci. Rep. 2017, 7, 7432.

[12] S. C. L. Hall, C. Tognoloni, J. Charlton, E. C. Bragginton, A. J. Rothnie, P. Sridhar, M. Wheatley, T. J. Knowles, T. Arnold, K. J. Edler, T. R. Dafforn, Nanoscale 2018, 10, 10609.

[13] K. Yasuhara, J. Arakida, T. Ravula, S. K. Ramadugu, B. Sahoo, J. I. Kikuchi, A. Ramamoorthy, J. Am. Chem. Soc. 2017, 139, 18657.

[14] A. O. Oluwole, B. Danielczak, A. Meister, J. O. Babalola, C. Vargas, S. Keller, Angew. Chem., Int. Ed. 2017, 56, 1919.

[15] F. Vial, A. G. Oukhaled, L. Auvray, C. Tribet, Soft Matter 2007, 3, 75.

[16] Y. Gohon, F. Giusti, C. Prata, D. Charvolin, P. Timmins, C. Ebel, C. Tribet, J. L. Popot, Langmuir 2006, 22, 1281.

[17] J. L. Popot, T. Althoff, D. Bagnard, J. L. Baneres, P. Bazzacco, E. Billon-Denis, L. J. Catoire, P. Champeil, D. Charvolin, M. J. Cocco, G. Cremel, T. Dahmane, L. M. de la Maza, C. Ebel, F. Gabel, F. Giusti, Y. Gohon, E. Goormaghtigh, E. Guittet, J. H. Kleinschmidt, W. Kuhlbrandt, C. Le Bon, K. L. Martinez, M. Picard, B. Pucci, J. N. Sachs, C. Tribet, C. van Heijenoort, F. Wien, F. Zito, M. Zoonens, Annu. Rev. Biophys. 2011, 40, 379. 